\title{
Análisis topológico mediante el Método de Elementos Finitos del chasis de una motocicleta de competencia
}

\section{(Topological analysis using the Finite Element Method of the chassis of a competition motorcycle)}

\begin{abstract}
Álvaro Remache ${ }^{1}$, Julio Leguisamo², Edwin Tamayo ${ }^{3}$
Resumen

En este estudio se implementó una técnica de optimización topológica en el bastidor de una motocicleta de competencia Honda CRF 230, con el objetivo de reducir el peso del componente original manteniendo los límites de seguridad de las propiedades mecánicas del material, para obtener un mayor rendimiento del motor y mayor maniobrabilidad para el piloto en una competencia. Para ello, se efectuó la caracterización del material original del chasis de la motocicleta mediante un procedimiento llamado Espectrometría de Chispa, además se realizó el análisis estático del chasis para determinar las condiciones de frontera para la simulación. Para el desarrollar el CAD del chasis y la simulación se utilizó el SolidWorks Simulation. La aplicación de la optimización topológica se realizó utilizando un criterio de distribución de esfuerzos, por medio de la utilización del método de MEF. Finalmente, se analizó la estructura original frente a la modificada para obtener como resultado una reducción del $67.6 \%$ de peso del chasis original, con un factor de seguridad de 1.41 .
\end{abstract}

\section{Palabras clave}

Simulación; optimización topológica; elementos finitos; materiales; propiedades mecánicas.

\begin{abstract}
This model presents a technical application of topological optimization, applied through the study of the behavior of the frame of a brand competition motorcycle, Honda CRF 230 model, with the aim of reducing the weight of the original component without altering its mechanical properties, and obtain a higher performance of the engine, greater maneuverability for the pilot and the reduction of manufacturing costs. This method of analysis is used to obtain the percentages of the chemical elements of a piece by the intensity of the light emitted by a spark, thereby acquiring the mechanical properties of the material. By means of the results, they are analyzed in different points of stress concentration of the frame; the conditions of the border are set to establish the simulation in the CAD model, and the application of the optimization of the structure through the use of the MEF method. Finally, the reduction of $67.6 \%$ of the weight of the original chassis was included, with a safety factor of 1.41.
\end{abstract}

\section{Keywords}

Simulation; Topological Optimization; Finite elements; Materials; Mechanical properties.

\section{Introducción}

El análisis mediante el método de elementos finitos, según lo manifiestan Naula, Albuja, Carrillo, e Izurieta (2016), es la vía para dar solución a los problemas de ingeniería, caracterizado por el estudio de modelos matemáticos abstractos, en los cuales intervienen un conjunto de parámetros para predecir fallos en todo sistema físico continuo con un material específico (Vinueza y Gutiérrez, 2018). Xiao, Liu, Du, Wang, y He (2012), lo definen como un método para dar solución a varias incógnitas que se presentan en la ingeniería, donde la resolución involucra varios 
parámetros de dificultad, cuando se trabaja con geometrías complejas, cargas no distribuidas, cuando están en función de las propiedades de los materiales y porque los resultados analíticos generalmente requieren la solución de ecuaciones diferenciales parciales u ordinarias que no son posibles de resolver (Cubillos, 2015).

La optimización de la topología es una práctica de ingeniería bien establecida para optimizar el diseño de las piezas y crear estructuras livianas y de bajo costo; que históricamente han sido difíciles o imposibles de realizar (Neches, 2015). En el estudio de Meza, Tamayo y Franco (2015), indica que la optimización topológica permite la síntesis de estructuras con valores óptimos de varios de sus parámetros físicos. En relación con las partes mecánicas de bajo peso, implican menores costos por material y menor consumo de combustible en el caso de vehículos de transporte (Silvestre y Robles 2017). En general, la reducción de la inercia en partes de movimiento sea maquinaria o vehículos disminuye la cantidad de energía necesaria para su operación (Powar, Joshi, Khuley y Yesane, 2016).

Se han elaborado análisis topológicos en diversos trabajos utilizando diferentes metodologías para innumerables tipos de piezas mecánicas, que son utilizadas tanto en el área automotriz como industrial y en trabajos de chasis de motocicletas (Powar, Joshi, Khuley, y Yesane 2016), y de la misma manera para el diseño de reducción de material en chasis automotrices Mantovani, Campo, Ferrari, y Cavazzuti (2018). Mediante la utilización de un algoritmo de Meza, Tamayo y Franco (2015), que se usó para rediseñar una pieza mecánica existente, donde la optimización topológica permitió reducción del peso de un 24 \%, o el caso de estudio de Ramírez, López y Salazar (2013), que utiliza una metodología donde el área de estudio es un soporte de balancín utilizado en suspensiones automotrices, para minimizar el uso del material, donde se realiza la modificación geométrica de la estructura, utilizando el criterio de distribución de esfuerzos homogéneos permitió minimizar el volumen al 35 \% de la estructura inicial, en el caso de Ochoa y Salameda (2013), utiliza la caracterización a través de un estudio de cargas estáticas y dinámicas a partir de resultados y simulaciones computacionales de una pieza automotriz donde se logra reducir el peso del material a un $17.3 \%$.

El interés del estudio se centra en la importancia que tiene el peso de las autopartes y el significado que representa en el medio de las competencias, en este caso Reyes (2017) argumenta que toda motocicleta que esté relacionada en cualquier tipo de carrera debe contar con un alto rendimiento en términos de aceleración, frenado, manejo y principalmente peso Wang, J., Zhou, J., Li, B. B., Li, X. L., y Song, S. Y. (2012). Además, debe ser lo suficientemente resistente para completar con éxito las etapas descritas de una competencia, los factores de diseño que deben ser considerados tales como: estática, costo, ergonomía, facilidad de mantenimiento, fabricación y fiabilidad.

Por lo expuesto en el presente estudió el objetivo de este trabajo fue determinar si al aplicar una optimización topológica mediante el método de elementos finitos en un chasis de motocicleta de competencia honda CFR230 en condiciones estáticas, los valores de tensión, desplazamiento, deformación unitaria y coeficiente de seguridad están dentro de la tolerancia del material original del chasis. Para lo cual se caracterizará el material del chasis mediante una espectrometría de chispa, se establece el modelo matemático para el análisis estático, se modela y se simula el chasis mediante el software Solid Works Simulation y finalmente se analiza los resultados obtenidos entre el chasis sin modificaciones y el modificado. 


\section{Metodología}

El análisis topológico se aplica a diferentes tipos de piezas mecánicas, se ha utilizado en el área aeroespacial, automotriz y obras de infraestructura (Silvestre y Robles, 2017), este trabajo está enfocado en el campo de las competencias, considerando que esta disciplina cada vez tiene más fuerza en nuestro país. El objetivo es optimizar el diseño del chasis de una motocicleta de competencia con un mínimo de peso en comparación al original, de esta manera sea aprovechado al máximo por el piloto.

En lo que se refiere al alcance de este trabajo se lo ha denominado de tipo experimental ya que estudia el problema de manera controlada para una obtención de resultados y su análisis posterior (Bernal, 2016). En este estudio se ha efectuado el análisis del bastidor de una motocicleta de marca y modelo honda CRF 230, de acuerdo con las especificaciones técnicas que se detallan en la Tabla 1, estos son utilizados para el cálculo del análisis estático, de manera específica en valores de dimensiones y pesos, en lo posterior con los resultados obtenidos se podrá establecer las condiciones de frontera, para la elaboración de las simulaciones computacionales.

Tabla 1. Especificaciones técnicas de la motocicleta

\begin{tabular}{|c|c|}
\hline \multicolumn{2}{|c|}{ ESPECIFICACIONES TÉCNICAS MOTOCICLETA HONDA CFR230 } \\
\hline Motor & Mono cilíndrico 4 tiempos \\
\hline Refrigeración & Aire \\
\hline Cilindrada & $223 \mathrm{cc}$ \\
\hline Potencia máxima & 13.7 KW /8.000 r.p.m. \\
\hline Encendido & Descarga del condensador \\
\hline Arranque & Eléctrico \\
\hline Transmisión & 6 velocidades \\
\hline Chasis & Cuna semi-doble, tubo de acero \\
\hline Avance & $112 \mathrm{~mm}$ \\
\hline Lanzamiento & $27^{\circ} 18^{\prime}$ \\
\hline Suspensión. delantera & Horquilla telescópica \\
\hline Suspensión. trasera & Basculante con ajuste de precarga \\
\hline Freno delantero & Disco Hidráulico Ø 240 mm x 3 mm \\
\hline Freno trasero & $110 \mathrm{~mm}$ \\
\hline Longitud & $2059 \mathrm{~mm}$ \\
\hline Ancho & $815 \mathrm{~mm}$ \\
\hline Altura & $1159 \mathrm{~mm}$ \\
\hline Distancia entre ejes & $1375 \mathrm{~mm}$ \\
\hline Altura al asiento & $865 \mathrm{~mm}$ \\
\hline Distancia libre al suelo & $300 \mathrm{~mm}$ \\
\hline Capacidad del tanque & 7.2 litros \\
\hline
\end{tabular}

Fuente: Honda (2013). 


\section{1 Caracterización del material}

El elemento que se emplea para la elaboración del estudio, es un material compuesto, según se detalla en la Tabla 2, para la caracterización del material se ha elaborado la investigación en los laboratorios del Departamento de Metalurgia extractiva de la Escuela Politécnica Nacional del Ecuador, utilizando una metodología que trata de un análisis por Espectrometría de Chispa, específicamente se ha utilizado el equipo de denominación Espectrómetro de Marca BRUKER modelo Q4TASMAN, donde se detallan los datos con cifras decimales del porcentaje de cada elemento químico que compone el material.

Al revisar la bibliografía que proporciona Pino (2002), obtenemos el listado de aceros de bajo carbono, en la Tabla 3 , se encuentra el material que cumple con los requerimientos químicos aproximados en comparación con los porcentajes químicos del material inicial de la Tabla 2.

Tabla 2. Caracterización material

\begin{tabular}{|l|c|}
\hline \multicolumn{1}{|c|}{ Metales } & Chasis de motocicleta (\%) \\
\hline Carbono(C) & 0.209 \\
\hline Silicio (Si) & 0.163 \\
\hline Manganeso (Mn) & 0.912 \\
\hline Cromo (Cr) & 0.018 \\
\hline Molibdeno (Mo) & 0.005 \\
\hline Níquel (Ni) & 0.009 \\
\hline Cobre (Cu) & 0.010 \\
\hline Aluminio (Al) & 0.027 \\
\hline Cerio (Ce) & 0.160 \\
\hline Plomo (Pb) & 0.018 \\
\hline Wolframio (W) & 0.300 \\
\hline Hierro (Fe) & 98.08 \\
\hline
\end{tabular}

Fuente: ESPN (2018).

Tabla 3. Composición quimica del acero SAE 8620

\begin{tabular}{|l|l|l|l|l|l|l|}
\hline \multicolumn{7}{|c|}{ ANÁLISIS TíPICO \% } \\
\hline & \multicolumn{1}{|c|}{ C } & \multicolumn{1}{|c|}{ SI } & \multicolumn{1}{c|}{ Mn } & \multicolumn{1}{c|}{ Ni } & \multicolumn{1}{c|}{ Cr } & \multicolumn{1}{c|}{ Mo } \\
\hline Material & 0.21 & 0.163 & 0.912 & 0.009 & 0.018 & 0.005 \\
\hline SAE 8620 & $0.17-0.23$ & $0.10-0.40$ & $0.60-0.95$ & $0.35-0.75$ & $0-35-75$ & $0.15-0.25$ \\
\hline
\end{tabular}

Fuente: ESPN (2018).

En la Tabla 4 se visualizan las propiedades mecánicas y tecnológicas del material que se va a utilizar, es un SAE 8620 (Aceros al Cromo, Níquel y Molibdeno).

Las propiedades mecánicas de un material son las que se encuentran relacionadas cuando el elemento se somete a esfuerzo, además de la importancia de la utilización de materiales compuestos (Airoldi, A., Bertoli, S., Lanzi, L., Sirna, M., y Sala, G., 2012). En esta investigación para la elaboración de simulaciones se va a trabajar con los valores observados en la Tabla 4, son las 
caracteísticas mesurables capaces de calificar un comportamiento o una respuesta del mismo a situaciones externas, independientemente del tamaño y la geometría del elemento considerado.

Tabla 4. Propiedades Mecánicas del Acero 8620

\begin{tabular}{|l|l|l|}
\hline \multicolumn{1}{|c|}{ PROPIEDADES } & \multicolumn{1}{c|}{ MÉTRICO } & \multicolumn{1}{c|}{ IMPERIAL } \\
\hline Resistencia a la tracción & $530 \mathrm{MPa}$ & $76.900 \mathrm{psi}$ \\
\hline Resistencia a la fluencia & $385 \mathrm{MPa}$ & $55.800 \mathrm{psi}$ \\
\hline Módulos elásticos & $190-210 \mathrm{GPa}$ & $27557-30458 \mathrm{ksi}$ \\
\hline Densidad de la masa & $7840 \mathrm{~kg} / \mathrm{m}^{3}$ & \\
\hline Módulo de compresibilidad (típico para el acero) & $140 \mathrm{GPa}$ & $20300 \mathrm{ksi}$ \\
\hline Módulo de cizallamiento (típico para el acero) & $80 \mathrm{GPa}$ & $11600 \mathrm{ksi}$ \\
\hline Coeficiente de Poisson & $0,27-0,30$ & $0,27-0,30$ \\
\hline Impacto Izod & $115 \mathrm{~J}$ & $84.8 \mathrm{ft} . \mathrm{lb}$ \\
\hline Dureza, Brinell & 149 & 149 \\
\hline Dureza, Knoop (conversión de dureza Brinell) & 169 & 169 \\
\hline Dureza, Rockwell B (convertido a partir de la dureza Brinell) & 80 & 80 \\
\hline Dureza, Vickers (conversión de dureza Brinell) & 155 & 155 \\
\hline Maquinabilidad & 65 & 65 \\
\hline
\end{tabular}

Fuente: Astm (2018).

\subsection{Cálculo de análisis estático}

Para establecer las condiciones de frontera y elaborar las simulaciones se necesita los resultados de un análisis estático. Este cálculo se realiza mediante el diagrama de cuerpo libre, con este gráfico se identifica la magnitud y ubicación de las fuerzas que actúan en el bastidor, en la Figura 1 (a) y (b) detalla la ubicación y dirección de las magnitudes físicas, estas se identifican mediante una referencia en la revisión bibliográfica de Ochoa y Salameda (2013), este estudio aporta con ecuaciones matemáticas para hallar los valores de los porcentajes de cargas en los ejes delantero y posterior, también los esfuerzos que se encuentran ubicados en cada uno de los puntos establecidos del chasis.

Figura 1. Ubicación de dimensiones y fuerzas en los puntos estratégicos del chasis

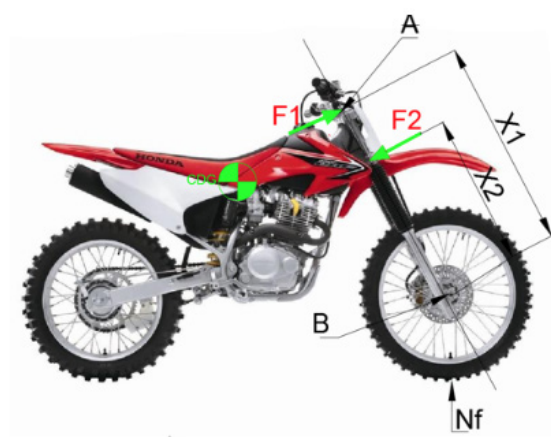

(a) -Horquilla

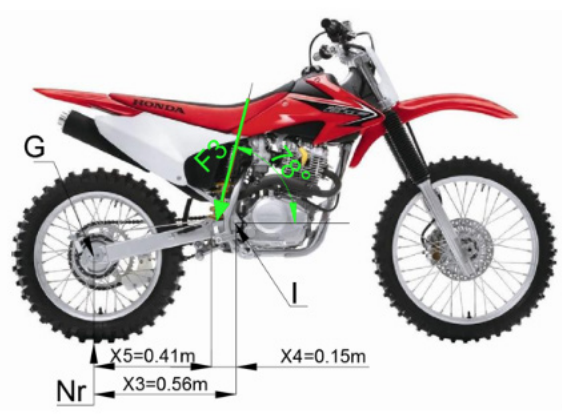

(b) -Oscilante 
En el diagrama de cuerpo libre que se observa en la Figura 2 (a) y (b), muestra la ubicación y la descomposición de fuerzas, magnitudes físicas y distancias de cada punto en el chasis, se lo ha dividido como horquilla y oscilante.

Figura 2. Descomposición de fuerzas y distancias de la horquilla y el oscilante

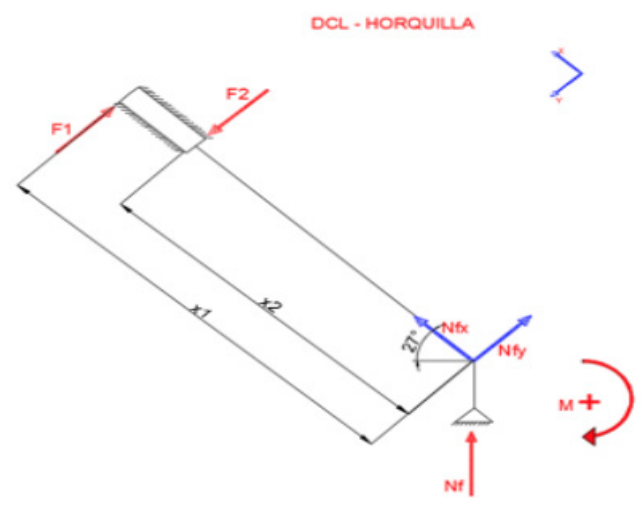

(a) - Horquilla

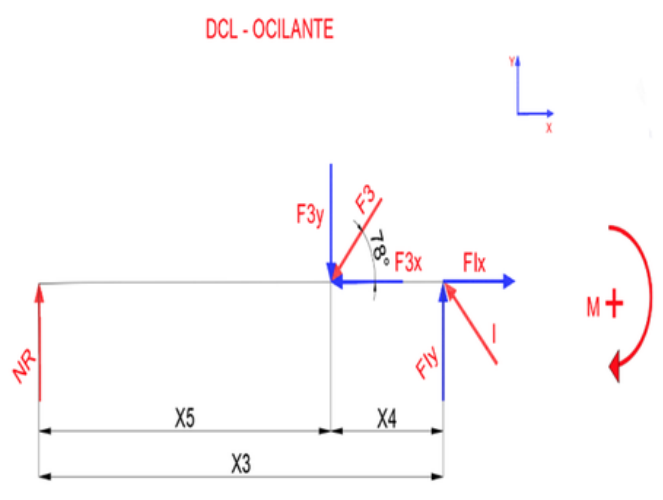

(b) - Oscilante

Es indispensable determinar los porcentajes de cargas en los ejes delantero y posterior ya que son necesarios para hallar los resultados de las fuerzas normales con respecto al suelo y el peso de la moto con el ocupante (hombre), a través del análisis estático, para esto se considera un listado de ecuaciones.

Porcentaje de carga estático en el eje delantero.

$$
\operatorname{ced}=\frac{p-b}{p} \cdot 100 \%
$$

Porcentaje de carga estático en el eje posterior.

$$
\text { cep }=100-\text { ced }
$$

Valores de la fuerza normal (eje delantero y posterior) con respecto al suelo y el peso de la moto y el ocupante.

$$
\begin{aligned}
& \mathrm{Nf}=(w m t+w p) \cdot c e d p \\
& \mathrm{Nr}=(w m+w p) \cdot c e p p
\end{aligned}
$$

Donde:

p Distancia entre ejes

b Distancia del CG y eje delantero

wp Masa ocupante (hombre)

wm Masa de la Moto

cedp \% de carga eje delantero

cepp \% de carga eje posterior

Nf Normal eje delantero

$\mathrm{Nr} \quad$ Normal eje posterior 
Para determinar los valores del análisis estático, y las fuerzas que actúan sobre los puntos de concentración de esfuerzos se consideran los siguientes cálculos generales (ecuación 4 - 9)

Ecuación para determinar los valores de F1 es la siguiente

$$
F 1=\frac{(N f y-X 2)}{(X 1-X 2)}
$$

Ecuación para determinar los valores de F2 es la siguiente

$$
\mathrm{F} 2=\mathrm{NFy}+\mathrm{F} 1
$$

Ecuación para determinar los valores de F3 es la siguiente

$$
F 3=\frac{F 3 y}{0.7814}
$$

Ecuación para hallar el momento máximo.

$$
\text { Mmáx }=F 1(0.18 m)
$$

Ecuación para hallar Momento de inercia.\}

$$
I=\left[\left(D^{4}-d^{4}\right) \frac{\pi}{64}\right]
$$

Ecuación para hallar el esfuerzo máximo de deformación.

$$
\text { Emáx }=\frac{\text { Mmax.r }}{\mathrm{I}}
$$

Donde:

$\mathrm{X} 1 \quad$ Distancia 1 chasis

$\mathrm{X} 2 \quad$ Distancia 2 chasis

F1 Fuerza 1 Horquilla

F2 Fuerza 2 Horquilla

F3 Fuerza 3 Oscilante

Mmáx Momento máximo

I Momento de inercia

Emax

Esfuerzo máximo de deformación

\subsection{Chasis CAD}

Para ejecutar las simulaciones, se ha elaborado la geometría en un programa CAD, por medio de un levantamiento dimensional del bastidor original estándar, el modelo ha sido creado en el software computacional de SolidWorks Simulation, como se puede apreciar en la Figura 3. 
Figura 3. Modelo de chasis Honda "CRF 230" SolidWorks

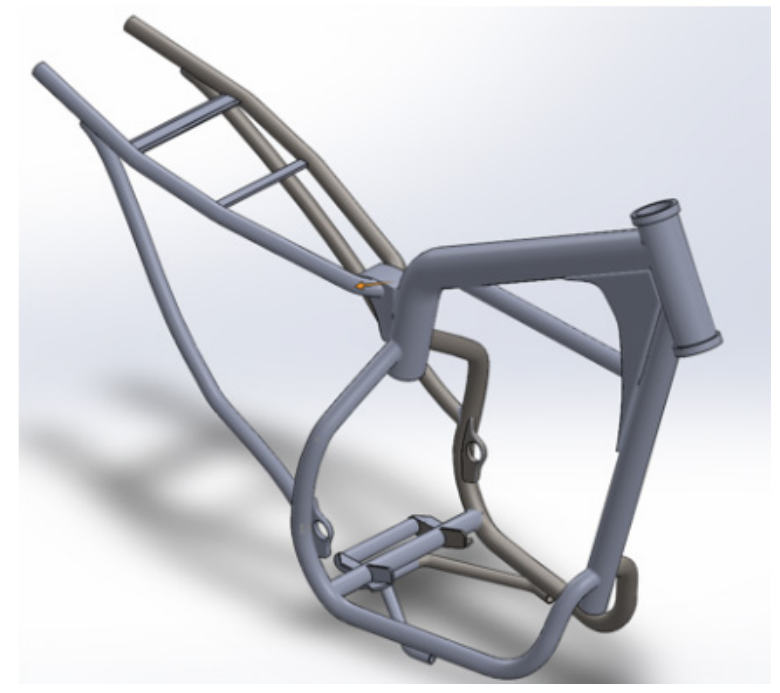

\subsection{Simulación y análisis por elementos finitos}

\subsection{Aplicación de materiales, cargas y soportes}

En el proceso de elaboración de las simulaciones se elige el material especificado a todo el cuerpo geométrico para luego aplicar las cargas y soportes en los puntos calculados anteriormente mediante los resultados del análisis estático, se utiliza el sistema de cargas puntuales, en lugares estratégicos del chasis, donde se generan las mayores localidades de esfuerzos, Figura 4.

Figura 4. Muestra el sistema de coordenadas, y la aplicación de fuerzas verticales y transversales, así como los puntos fijos, en puntos específicos del bastidor donde se efectuará el análisis, Fuerza $1=2596.989 \mathrm{~N}-$ Fuerza 3= 3294.688 N - Fuerza $4=4003.634 \mathrm{~N}$

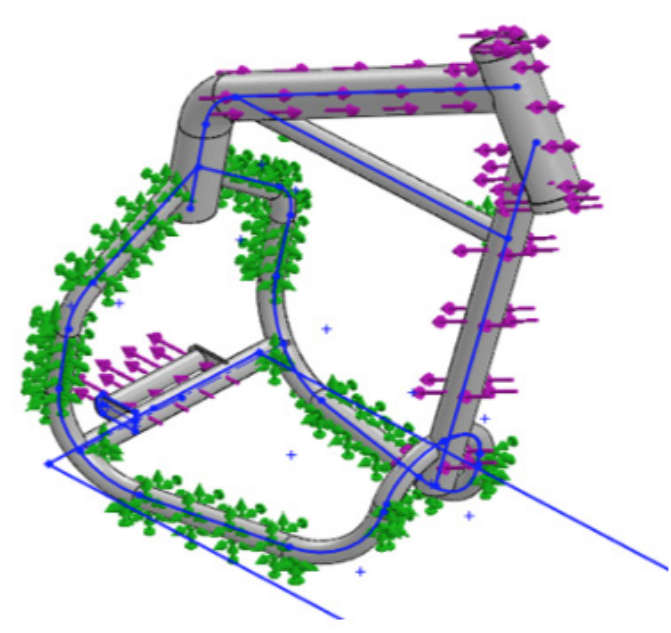

1. Sistema de coordenadas

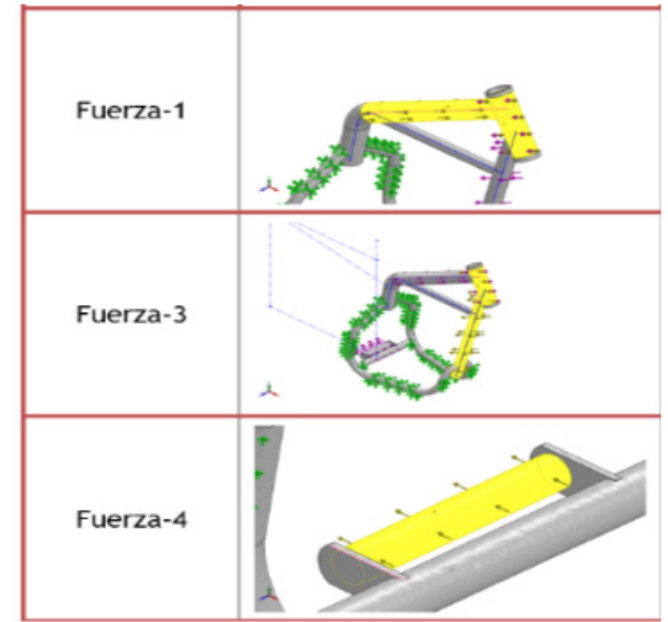

2. Aplicación de fuerzas verticales y transversales 


\subsubsection{Selección del mallado}

Debido a que la convergencia de la solución depende de la calidad de la malla, es necesario hacer un buen uso de las herramientas de mallado y evitar problemas con el peso computacional (Álvarez, R., Benito, J., Ureña, F., Salete, E., y Aranda, E., 2016). Para el criterio de mallado y generación de elementos de discretización del dominio a través de esta geometría, se ha utilizado un mallado triangular fino, esto garantiza la obtención de datos lo suficientemente precisos para el análisis posterior, observar la Figura 5.

Figura 5. Configuración general del mallado para el bastidor, Número total de nodos = 96 938, Número total de elementos $=49180,00 \%$ de elementos cuyo cociente de aspecto es $<3=23.1$, $\%$ de elementos cuyo cociente de aspecto es $>10=0.525$

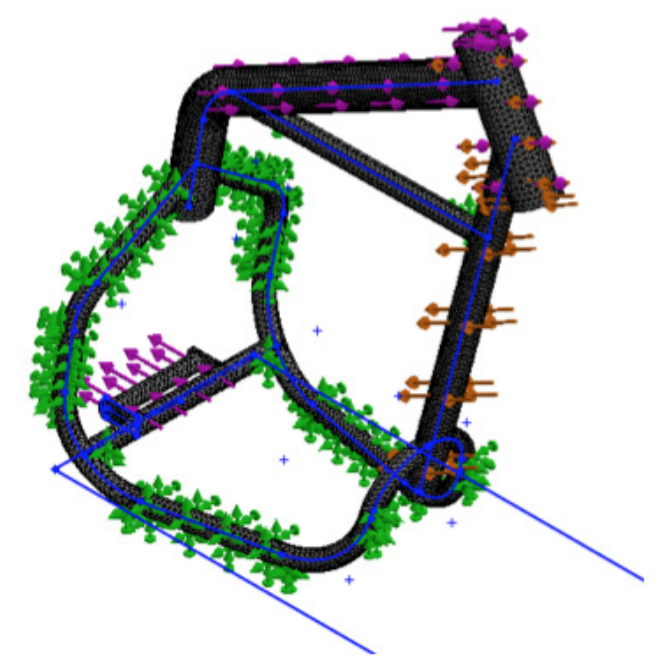

\section{Resultados y discusión}

En la Tabla 5 se observan las consideraciones generales de los porcentajes de carga delantero y posterior del bastidor que se ejecutó mediante las ecuaciones (1-2), adicionalmente se obtiene los resultados de las fuerzas normales de los ejes calculados mediante las ecuaciones (3), estos valores se utilizan para hallar las fuerzas que actúan en los puntos de concentración de esfuerzos del chasis.

Tabla 5. Fórmulas y valores de consideraciones generales del análisis estático

\begin{tabular}{|c|c|c|c|c|}
\hline $\begin{array}{c}\text { Carga estática eje } \\
\text { delantero } \\
(\%)\end{array}$ & $\begin{array}{c}\text { Carga estática eje } \\
\text { Posterior } \\
(\%)\end{array}$ & $\begin{array}{c}\text { Peso moto } \\
\text { y ocupante } \\
(\mathbf{N})\end{array}$ & $\begin{array}{c}\text { Normal eje } \\
\text { Delantero } \\
(\mathbf{N})\end{array}$ & $\begin{array}{c}\text { Normal eje } \\
\text { Posterior } \\
(\mathbf{N})\end{array}$ \\
\hline 0.43 & 0.57 & 1833.8 & 783.0 & 1048.9 \\
\hline
\end{tabular}

La Tabla 6 muestra los resultados de las fuerzas ubicadas en la horquilla y el oscilante que fueron obtenidos a través de las ecuaciones (5-9), estas variables ejercen en cada uno de los lugares donde se han considerado que existe mayor cantidad de concentración de esfuerzos. Los resultados van de la mano con los obtenidos en el estudio de Ochoa y Salameda (2013), 
el comportamiento de las fuerzas aplicadas según los valores obtenidos determinan como está sometido el bastidor en relación al peso del ocupante y de la máquina.

Tabla 6. Resultados de cálculos relacionados a la horquilla y oscilante

\begin{tabular}{|c|c|c|c|c|c|c|c|c|c|}
\hline $\begin{array}{l}\text { Valores } \\
\text { calcula- } \\
\text { dos }\end{array}$ & $\begin{array}{l}\mathrm{X} 1 \\
(\mathrm{~m})\end{array}$ & $\begin{array}{c}X 2 \\
\text { (m) }\end{array}$ & $\begin{array}{l}\mathrm{Nfx} \\
\text { (N) }\end{array}$ & $\begin{array}{l}\text { Nfy } \\
\text { (N) }\end{array}$ & $\begin{array}{l}\text { F1 } \\
\text { (N) }\end{array}$ & $\begin{array}{l}\text { F2 } \\
\text { (N) }\end{array}$ & $\begin{array}{c}\text { Momento } \\
\text { Máximo } \\
(\mathrm{Nm})\end{array}$ & $\begin{array}{l}\text { Momento } \\
\text { Inercia } \\
(\mathrm{Nm})\end{array}$ & $\begin{array}{c}\text { Esfuerzo } \\
\text { Máximo } \\
\text { (MPa) }\end{array}$ \\
\hline \multirow[t]{2}{*}{ Horquilla } & 0.85 & 0.67 & 355.4 & 697.7 & 2596.9 & 3294.6 & 467.45 & $5.589 \times 10=$ & 114.12 \\
\hline & $\begin{array}{l}\text { X3 } \\
\text { (m) }\end{array}$ & $\begin{array}{l}\mathrm{X} 4 \\
(\mathrm{~m})\end{array}$ & $\begin{array}{l}\text { X5 } \\
\text { (m) }\end{array}$ & $\begin{array}{l}\mathrm{Nr} \\
(\mathrm{N})\end{array}$ & $\begin{array}{l}\text { F3 } \\
\text { (N) }\end{array}$ & $\begin{array}{l}\mathrm{Fl} \\
(\mathrm{N})\end{array}$ & $\begin{array}{l}\text { Momento } \\
\text { Máximo } \\
(\mathrm{Nm})\end{array}$ & $\begin{array}{l}\text { Momento } \\
\text { Inercia } \\
(\mathrm{Nm})\end{array}$ & $\begin{array}{l}\text { Esfuerzo } \\
\text { Máximo } \\
\text { (MPa) }\end{array}$ \\
\hline Oscilante & 0.56 & 0.15 & 0.41 & 1048.9 & 4003.6 & 2985.5 & 430.07 & $2.129 \times 10^{3}$ & 323.06 \\
\hline
\end{tabular}

En la Figura 6 (a), se observa los puntos donde existe la mayor concentración de esfuerzos del chasis, en la aplicación de fuerzas tanto en la horquilla y el oscilante del bastidor los resultados que se muestran a través el módulo de von mises da un valor máximo es de 137.36 MPa, la Figura 6 (b) presenta la tabla de desplazamiento con un máximo de 0.062 mm, este valor que se encuentra dentro de las condiciones de diseño, también se considera que la gráfica refleja una escala de deformación de 1053.75.

Figura 6(a). Resultado de los valores de la simulación a través del módulo de Von mises, Tensiones 1, MíNIMO 1.00373e-014 N/mm² (MPa) - Nodo: 82308, MÁXIMO $137.52 \mathrm{~N} / \mathrm{mm}^{2}$ (MPa) - Nodo: 96389.
Figura 6(b). Valores resultantes de desplazamiento mediante el método de elementos finitos Desplazamientos1, MÍNIMO 0 mm - Nodo: 141, MÁXIMO 0.0615483 mm, Nodo: 108.
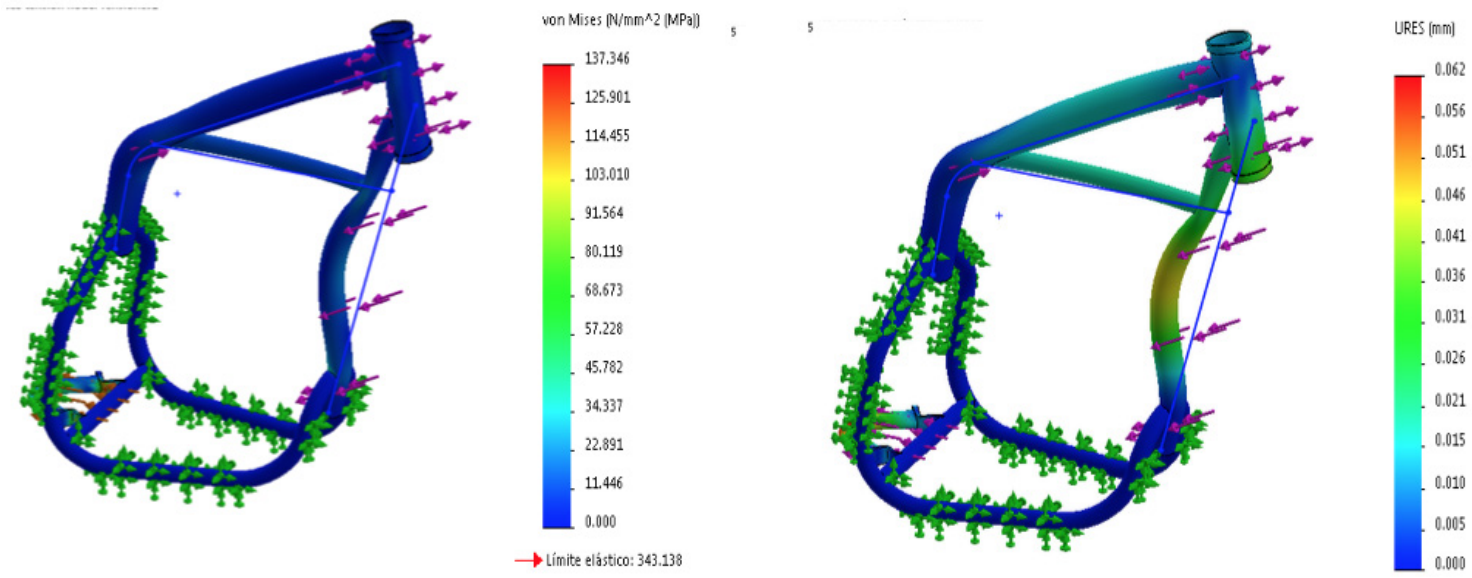

En la Figura 7, se visualiza la gráfica con los valores del factor de seguridad mediante la simulación, este resultado se determina a través de la división del límite elástico del material para el valor de esfuerzo máximo del módulo de von mises, haciendo un análisis en cada uno de los nodos según un criterio de fallos, con un resultado de 2498. 
Figura 7. Resultado del factor de seguridad del chasis, Factor de seguridad1, MíNIMO 2.49518 - Nodo: 96389, MÁXIMO 1e+016 - Nodo: 1983
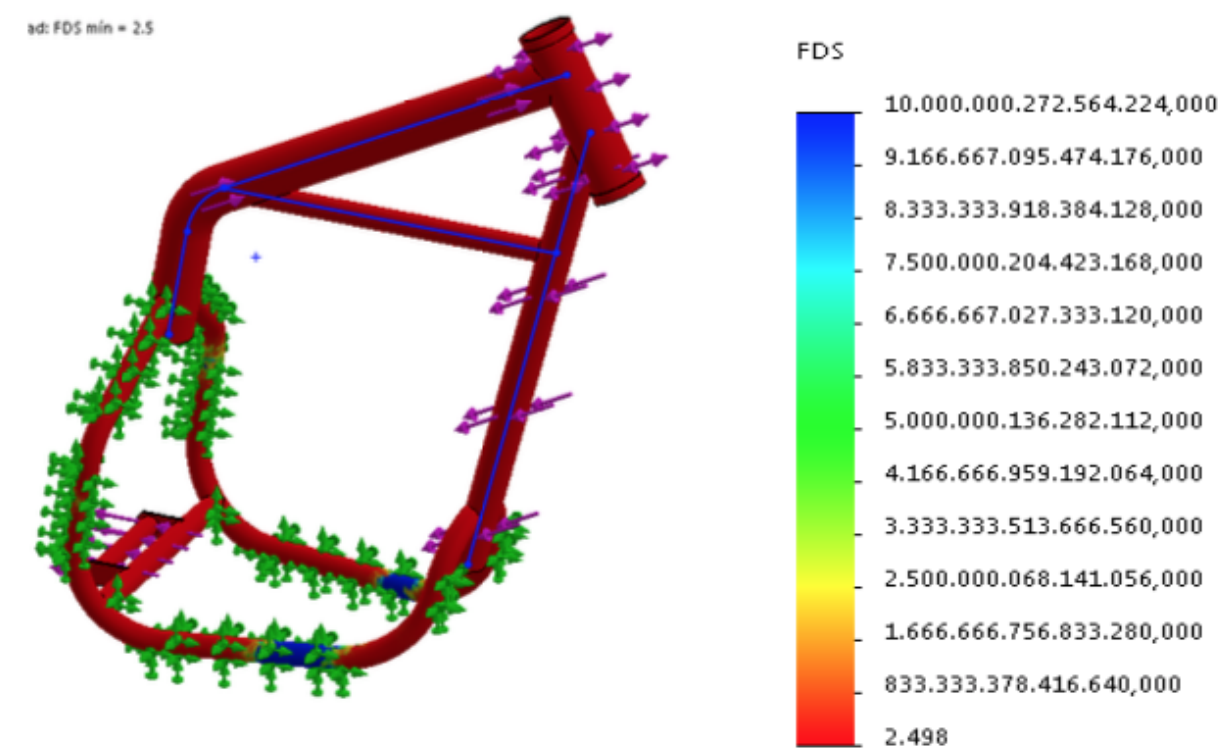

\subsection{Aplicación de la optimización topológica en el diseño del chasis}

Este método se aplica en base a un criterio de distribución de esfuerzos. El chasis original está diseñado para soportar una carga vertical en la base del amortiguador con un valor de 4003.63 $\mathrm{N}$, y dos cargas diagonales en diferentes sentidos en la horquilla de $2596.989 \mathrm{~N}$ y $3294.688 \mathrm{~N}$ respectivamente, el material del componente es un Acero de bajo carbono SAE 8620 que cuenta con un límite de fluencia de 343.13 MPa. Usando el programa SolidWorks Simulation, permitió calcular el volumen y la masa del componente inicial antes de la optimización con un valor de $4074.02 \mathrm{~g}$.

Para la aplicación del método de optimización topológica se utilizó una malla fina de 3.7 mm por nodo y con un tamaño de elementos de 608906 mm, a este componente se utilizó un dominio triangular a toda la pieza donde un milímetro equivale a un elemento finito, es decir, tiene una discretización con un total de 97176 elementos finitos (nodos). El dominio posee zonas no optimizables, estas estas ubicadas en las tuberías de menor diámetro de 19 mm que se encuentran ubicadas donde iría el asiento para el piloto.

La topología obtenida se muestra en la Figura 8, sin embargo, la estructura que se obtiene del análisis se muestra de forma difusa, por el motivo que la reducción de material se realizó de forma interna al espesor de los tubos, donde se requiere la interpretación de la geometría para utilizarla en un proceso convencional de diseño mecánico Scappaticci, L., Bartolini, N., Guglielmino, E., y Risitano, G. (2017). 
Figura 8. Estructura generada por optimización topológica, propiedades volumétricas como: Masa:2.76567 kg, Volumen:0.000352764 m3, Densidad:7840 kg/m3, Peso:27.1036 N

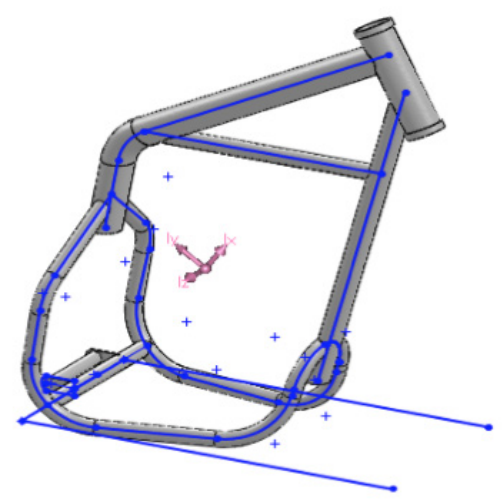

Propiedades de masa de CRF 230

Configuración Predeterminado (Como mecanizada)

Sistema de coordenadas - Predeterminado -

Densidad $=0.01$ gramos por milímetro

Masa $=2765.67$ gramos

Volumen $=352764.12$ milímetros cúbicos

Área de superficie $=555961.02$ milímetros cuadrados .

\author{
Centro de masa $=($ milímetros $)$ \\ $X=184.47$ \\ $Y=217.43$ \\ $\mathrm{Z}=0.13$
}

Para verificar que las dos estructuras están dentro de los parámetros de carga (original y optimizado), se ha considerado una comparación de valores con un análisis de distribución esfuerzos mediante el método de elementos finitos con el software comercial SolidWorks, en la Figura 9 (a) y (b) muestran la distribución del esfuerzo equivalente del módulo de von mises a través de todo el componente original y el componente aplicado la optimización topológica respectivamente. Donde el valor máximo equivalente de la distribución de esfuerzos de Von Mises para el chasis original tiene un valor de 137.346 MPa. Se observa además que las diversas articulaciones del componente donde indican valores de esfuerzos mínimos, eso representa el material de sobra, el factor de seguridad para el componente original es de 2.498 .

Figura 9. Esfuerzo equivalente de Von mises del chasis, a) Original Tensiones 1, MÍNIMO 1.00373e-014 N/mm² (MPa), Nodo: 82308, MÁXIMO $137.52 \mathrm{~N} / \mathrm{mm}^{2}$ (MPa), b) rediseño aplicado optimización topológica., Tensiones1, MíNIMO, $0 \mathrm{~N} / \mathrm{mm}^{2}(\mathrm{MPa})$ Nodo: 878, MÁXIMO $243.259 \mathrm{~N} / \mathrm{mm}^{2}$ (MPa) - Nodo: 84844

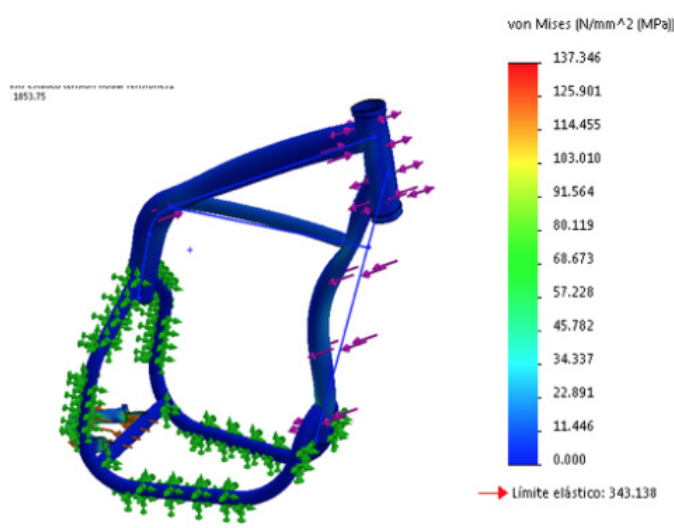

(a)

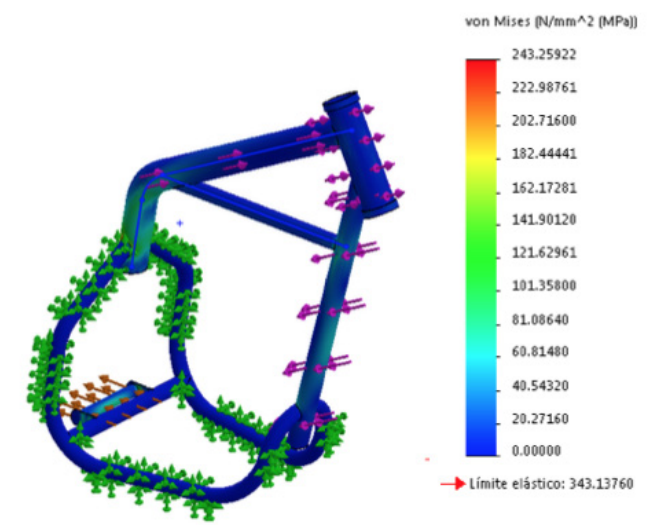

(b)

El valor del esfuerzo máximo de deformación del chasis aplicando la optimización topológica tiene un valor de $243.25 \mathrm{MPa}$, estas cargas están ubicadas en lugares estratégicos del chasis, cuenta con un factor de seguridad mínimo de 1.41. La mayor cantidad de material fue 
removido en las articulaciones del chasis que tienen un diámetro extenso, en esas zonas presentan valores de esfuerzos muy bajos, como se observa en la Figura 10 de tonalidad azul, es ahí donde se puede aplicar la optimización para que los esfuerzos calculados se encuentren dentro de los requerimientos mecánicos del material. Por ejemplo, en el elemento E1 que se tiene un valor inicial del $1.5 \mathrm{~mm}$ de espesor a comparación de su valor óptimo máximo resulto ser de 0.75 $\mathrm{mm}$, logrando distribuir la mayor cantidad de material en los lugares que presentan los valores de mayor esfuerzo. Como resultado final de la resultante de nuestro estudio se lo ha catalogado como satisfactorio ya que el valor inicial de masa del componente original es de $4074.02 \mathrm{~g}$ fue reducido a $2754.05 \mathrm{~g}$, por lo que se tiene un porcentaje de 67.6 \% de reducción.

Figura 10. Zonas donde se aplicó la optimización topológica

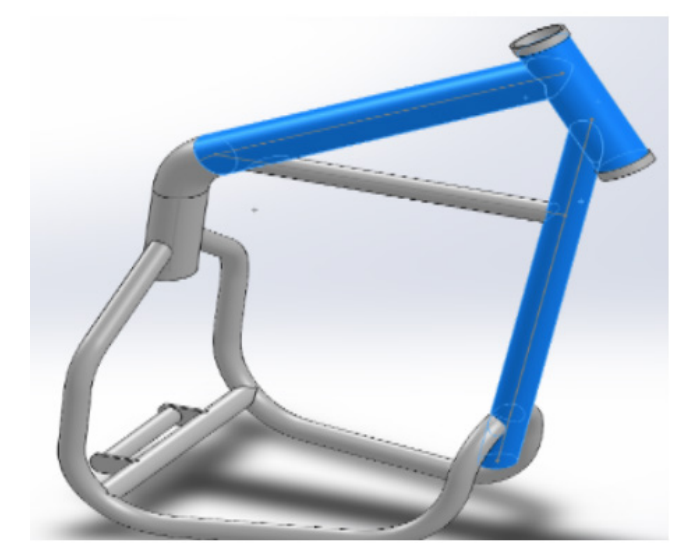

En la Figura 11, (a) y (b) se observa los Valores de esfuerzo equivalente de Von Mises del chasis original y el chasis optimizado haciendo la comparación de la distribución esfuerzos; obtenemos (a) un valor máximo de 137.346 MPa y en (b) el valor máximo es de 243.25 MPa, ligeramente mayor situado en el mismo punto de concentración del componete original, con la cantidad de material removido de la región del componente donde se presentan los valores de esfuerzo mas bajos.

Figura 11. Tabla de valores de esfuerzos equivalentes del chasis

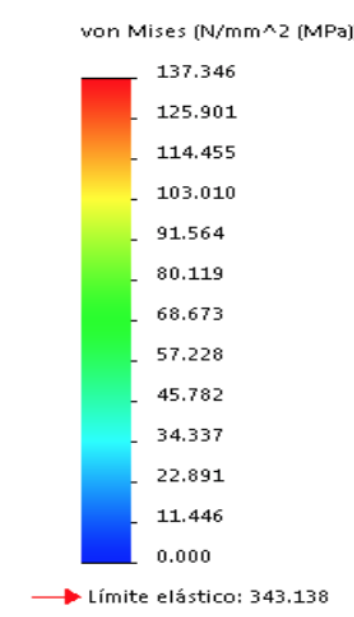

(a) Gráfica Original

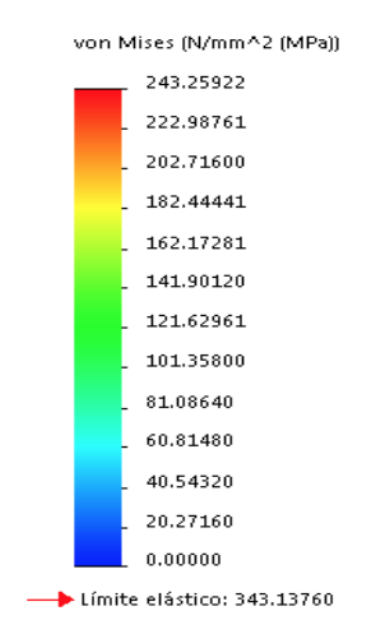

(b) Gráfica Optimizada 
La segunda parte del análisis es una comparación elaborada mediante el análisis del ingeniero David Vélez Leva en su trabajo de investigación titulado "Diseño, simulación y análisis de un chasis de moto para motor wankel", este estudio utiliza una metodología de optimización topológica a un chasis de motocicleta, donde se disminuye un 10 \% el tamaño de los tubos verticales del bastidor pasando de $25 \mathrm{~mm}$ a $18 \mathrm{~mm}$ en el diámetro exterior, en el diámetro interior a $22 \mathrm{~mm}$ a $15 \mathrm{~mm}$, una vez realizada el análisis del vom mises en los elementos seleccionados se confirma que la modificación es descartada por no satisfacer los criterios establecidos, al obtener un resultado de un aumento de tensión del módulo de von mises de 803.827 MPa a 1179.06 MPa ya que los valores de tensiones superan el valor del límite elástico del material, como se observa en la Tabla 7 se ha elaborado la comparación de los resultados de los dos estudios, en el cual se visualiza que, aplicado la misma metodología los valores encontrados en nuestro estudio están dentro de los criterios de diseño por que los resultados obtenidos están por debajo del valor del límite elástico del material, por otro lado en el estudio Vélez (2014), ha encontrado una solución mediante el diseño de un refuerzo entre los dos tubos verticales con el objetivo de disminuir los resultados de tensión, localizados en los valores extremos del travesaño hasta alcanzar un valor de $741.818 \mathrm{MPa}$ a diferencia del valor inicial de 803.827 MPa.

Tabla 7. Comparación de resultados experimentales basados en estudios realizados por otros autores. En la tabla se visualiza la comparación de los valores del estudio experimental vs los resultados basados en la investigación de Vélez (2014)

\begin{tabular}{|l|c|c|c|}
\hline & $\begin{array}{c}\text { Tensión - } \\
\text { Chasis original }\end{array}$ & $\begin{array}{c}\text { Tensión - Chasis } \\
\text { Optimizado }\end{array}$ & $\begin{array}{c}\text { Límite elástico } \\
\text { del material }\end{array}$ \\
\hline Vélez (2014) & $803.827 \mathrm{MPa}$ & $1020.27 \mathrm{MPa}$ & $460.00 \mathrm{MPa}$ \\
\hline Estudio Actual & $137.34 \mathrm{MPa}$ & $243.25 \mathrm{MPa}$ & $343.138 \mathrm{MPa}$ \\
\hline
\end{tabular}

En la norma del Instituto Ecuatoriano de Normalización (2009), en el punto 5 que trata sobre la Estructura de las Carrocerías, el párrafo 5.1.5.1, detalla que al existir una carga estática sobre la parte superior del elemento, equivale al 50 \% del peso máximo admisible para el chasis, distribuido uniformemente a lo largo del mismo, sin experimentar deformaciones en nungún punto; esto lo relacionamos con los valores de nuestro proyecto, las cargas máximas halladas mediante los resultados del programa se obtiene un valor de esfuerzo máximo hallado en el análisis topologico de $243.25 \mathrm{MPa}$ de tensión en el chasis, dividiriamos el limite elástico para el valor máximo de tensión se obtiene un valor de factor de seguridad que resulta 1.41 este valor se encuentra dentro de las tablas del factor de seguridad estático de Supp y Ort (2016), que se muestra en la Tabla 8, los valoremos mínimos de elementos y máquinas que estan sometidos a vibraciones y sin vibraciones, resultado la viabilidad de nuestra investigación.

Tabla 8. Valores de referencia del factor de seguridad estático

\begin{tabular}{|l|l|c|}
\hline \multicolumn{1}{|c|}{ Máquina Industrial G. } & \multicolumn{1}{|c|}{ Condiciones de Carga } & Límite más bajo de fs. \\
\hline \multirow{2}{*}{ Máquina Industrial } & Sin vibración ni impacto & 1.0 a 3.5 \\
\cline { 2 - 3 } & Con vibración o impacto & 2.0 a 5.0 \\
\hline \multirow{2}{*}{ Máquina Herramienta } & Sin vibración ni impacto & 1.0 a 4.0 \\
\cline { 2 - 3 } & Con vibración o impacto & 2.5 a 7.0 \\
\hline
\end{tabular}


Para el análisis de los resultados obtenidos de este proyecto se ha resumido en la Tabla 9, se elabora la comparación de valores del chasis original vs el chasis optimizado, garantizando la viabilidad del proyecto.

Tabla 9. Resumen de valores

\begin{tabular}{|l|l|l|}
\hline Componente & \\
\hline Modelo & & \\
\hline
\end{tabular}

\section{Conclusiones y recomendaciones}

El chasis de este estudio fue rediseñado con el propósito de eliminar el peso sobrante. Mediante el MEF computacional se generó una estructura óptima con una geometría un poco compleja que fue interpretada para obtener un modelo CAD de la pieza optimizada.

La optimización del diseño que se llevó a cabo en el chasis original consiguió la reducción de masa del elemento mediante la aplicación de un criterio de distribución de esfuerzos donde, el material que se ha eliminado ha sido considerado satisfactorio ya que se permitió calcular una reducción de $4074.02 \mathrm{~g}$ a $2754.05 \mathrm{~g}$, equivale al 67.6 \% de masa sin comprometer las propiedades mecánicas del material, el factor de seguridad del elemento optimizado que tiene un valor de 1.41, las condiciones de frontera se encuentran dentro de las especificaciones de diseño

El prototipo de chasis de moto se ha diseñado y modelado bajos los parámetros esperados ante las situaciones hipotéticas a las reales a través de los ensayos computacionales, evidenciando el comportamiento mecánico se ha obtenido las máximas tensiones del estudio 
con un valor de 137.34 MPa del prototipo inicial y de 243.25 MPa del prototipo modificado, resultados que se encuentran por debajo de los valores del límite elástico del material, garantizando un valor del factor de seguridad que se encuentra dentro de los criterios de diseño, frente a las cargas teóricas que se han aplicado sobre la estructura.

\section{Referencias}

Airoldi, A., Bertoli, S., Lanzi, L., Sirna, M., y Sala, G. (2012). Design of a motorcycle composite swing-arm by means of multi-objective optimisation. Applied Composite Materials, 19 (3-4): 599-618.

Álvarez, R., Benito, J., Ureña, F., Salete, E., y Aranda, E. (2016). Introducción al método de los elementso finitos. Universidad Nacional de Educación a Distancia, Madrid.Astm, S. (2018). SAE 8620. En. SAE 8620 STEEL Retrieved June 10, 2018, Consutado en http://www.astmsteel.com/product/ aisi-8620-steel-alloy/

Bernal, C. (2016). Metodología de la investigación. Administración, economía, humanidades y ciencias

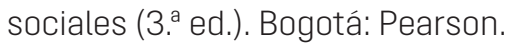

Cubillos, A. (2015). Introducción al método de elementos finitos. https://almec.files.wordpress. com/2007/10/resumen.pdf

ESPN. (2018). "Análisis químico por espectrometría de chispa." Quito - Ecuador.

Honda, M. (2018). HONDA Motorcycles. En Honda Manuales. Retrieved June 15, 2018, Consultado en http://honda.com.ec/moto/crf-230f/

Meza, C., Tamayo, F., y Franco, E. (2015). Optimización topológica aplicada al diseño de componentes estructurales mecánicos de peso reducido. El Hombre y la Máquina, 46: 71-78.

Naula, I., Albuja, G., Carrillo, A., e Izurieta, C. (2016). Modeling and numerical simulation of the Richards equation for infiltration problems. Enfoque UTE, 7 (1): 46-58.

Neches, L. (2015). Optimización topológica de problemas elásticos planos utilizando el método de elementos de contorno.Tesis de maestría, Facultad de mecánica Universidad de Sevilla, España.

Ochoa, J., y Salameda, A. (2016). Optimización del chasis de una motocicleta "KUNTUR", mediante el uso de un programa computacional "CAE". Tesis de Maestría, Facultad de Mecánica Universidad del Azuay.

Pino, A. (2015). Diagrama de Esfuerzo Deformación. En Monografías. Consultado June 26, 2018. Disponible en https:m.monografias.com/trabajos72/diagrama-esfuerzo-deformación/diagrama-esfuerzo-deformación.shtml

Powar, A., Joshi, H., Khuley, S., y Yesane, D. P. (2016). Analysis and Topological Optimization of Motorcycle Swing-Arm. INPRESSCO, 6.

Ramírez, F., López, E., y Salazar, C. (2013). Mejora estructural de un soporte tipo balancín a través de un análisis topológico. SOMIN, 5: 335-339.

Reyes, D. (2017). Diseño de las manguetas delanteras y posteriores para un vehículo Formula SAE mediante técnicas de optimización estructural y manufactura con CNC.Tesis de maestría, Facultad de mecánica, Universidad del Azuay. Cuenca, Ecuador.

Scappaticci, L., Bartolini, N., Guglielmino, E., \& Risitano, G. (2017). Structural optimization of a motorcycle chassis by pattern search algorithm. Engineering Optimization, 49 (8), 1373-1387.

Silvestre, C., y Robles, N. (2017). Diseño y Optimización Topológica de Rockers para un modelo de Formula Student. Tesis de Maestría, Facultad de mecánica,Escuela Técnica Superior de Ingeniería Universidad de Sevilla. Sevilla, España.

Supp, T. (2016). Cálculo del factor de seguridad estático. EE UU.

Budynas, R., y Nisbett, K. (2015). Diseño en ingeniería mecánica de Shigley (7.a ed.). ciudad de México, México: McGrawHill.

Vélez, L. (2014). Diseño, simulación y análisis de un chasis de moto para motor wankel. Tesis de Maestría, Facultad de Mecánica, Escuela Politécnica Superior de la Universidad Carlos III de Madrid. 
Vinueza, A., y Gutiérrez, R. (2018). Análisis por el método de elementos finitos del comportamiento de las pastillas de freno ABS con base de acero y zinc al discretizar el elemento continuo al utilizar "software" CAE. Enfoque UTE, 9 (1): 188-203.

Wang, J., Zhou, J., Li, B. B., Li, X. L., y Song, S. Y. (2012). The Optimization Design and Analysis of Motorcycle Frame Structure. Applied Mechanics and Materials 141: 569-573.

Xiao, D., Liu, X., Du, W., Wang, J., y He, T. (2012). Application of topology optimization to design an electric bicycle main frame. Structural and Multidisciplinary Optimization, 46 (6): 913-929. 